\title{
Study of Simple Correlation Coefficients for Yield and its Component Traits in Lentil (Lens culinaris Medik.)
}

\author{
Pankaj Kumar*, S.C. Vimal and Ajay Kumar \\ Department of Genetics and Plant Breeding, (Seed Technology Section), N.D. University of \\ Agriculture and Technology, Kumarganj, Faizabad 224 229, Uttar Pradesh, India \\ *Corresponding author
}

\section{A B S T R A C T}

An experiment was carried out during Rabi, 2015-17 at Students Instructional Form, Narendra Deva University of Agriculture and Technology, Narendra Nagar

Keywords

Pulse, Lentil,

Correlation coefficient.

\section{Article Info}

Accepted:

10 August 2017

Available Online:

10 September 2017 (Kumarganj), Faizabad (U.P.) to study the simple correlation coefficients among 13 quantitative characters with genotype NDL-1 in three replications using RBD Factorial design. Estimates of simple association coefficients among 13 quantitative characters estimated during 2015-16 and 2016-17 displayed that seed yield per plant exhibited highly positive significant associations with vigour index, biological yield per plant, seedling length, seed germination, harvest index, 1000 seed weight, number of seeds per pod, number of pods per plant, No. of branches/plant 60 days, No. of branches/plant 30 days, plant height $(\mathrm{cm}) 60$ days, plant height $(\mathrm{cm}) 30$ days, days to maturity and days to $50 \%$ flowering. All the other characters under study also displayed highly significant associations with each other besides seed yield per plant in both the years.

\section{Introduction}

The pulse is an annual leguminous crop yielding from one to twelve seeds of variable size, shape and color within a pod. Pulses are used for food for humans and other animals. Pulses are very important sources of protein in Indian diets as majority of population are vegetarian. Pulses are the third most important group of crops after cereals In the pulses includes; chickpea dry beans like pinto beans, kidney beans and navy beans; dry peas; and others among which lentil is a most important food legume. Lentil is eaten as Dahl, either whole or split. Lentil (Lens culinaris Medik) is of the important Rabi pulse, sown from October to December all over India either as a pure culture or as a mixture with sugarcane, linseed, rapeseed and mustard etc. The crop is harvested from February to April, depending upon the time of sowing. It can be grown on all kinds of soil. It can be successfully grown on poorer soil of moderate alkalinity. It can also tolerate drought. In the world India's rank $2^{\text {nd }}$ in respect of production as well as acreages followed by Turkey. In India it is grown in about $1.48 \mathrm{~m}$ ha with total production 1.03 mt, and productivity $697 \mathrm{Kg} / \mathrm{ha}$. Uttar Pradesh occupies $0.59 \mathrm{~m}$ ha area with 0.48 tones 
production and productivity $803 \mathrm{~kg} / \mathrm{ha}$., (Anonymous, 2013). Despite it is nutritional values and economic importance, chickpea production is relatively low in country; this is primarily due to poor genetic makeup of the cultivars available. Beside other reasons, the drought stress, poor management and biotic factors such as blight, wilt disease and pod borer are the major constraints to achieve potential yield. Yield is the major complicated traits that is an outcome of interaction of many plant characters and is highly influence by environmental changes. The direct selection of plant on seed yield basis may be misleading. Therefore, characters association (correlation) and path analysis must be studied to understand the contribution of genotype and environment towards the final yield before selection of plant.

\section{Materials and Methods}

The field experiments under present investigation were conducted during 2015-16 and 2016-17 at Student Instructional Farm and lab experiments were carried out in Seed Testing Laboratory of Seed Technology Section, N. D. University of Agriculture and Technology, Kumarganj, Faizabad (U. P.). Geographically, Narendra Nagar situated between $26.47^{\circ} \mathrm{N}$ latitude, $82.12^{\circ}$ longitude and at an altitude of 113 meters above the mean sea level. The climate of district Faizabad is semi-arid with hot summer and cold winter. Nearly 80 per cent of total rain fall is received during the monsoon the treatment details are presented in table 1 .

Plant to plant distance and row to row distance was 10 and $25 \mathrm{~cm}$, respectively. Fertilizer was applied @ 20:50:50 (kg ha $\left.{ }^{-1}\right)$ $\mathrm{N}: \mathrm{P}: \mathrm{K}$ at the time of sowing. The lentil crop was cultivated using standard agronomic practices. The observations were recorded at days to $50 \%$ flowering, Days to maturity, plant height $(\mathrm{cm})$ on 30 days, plant height (cm) on 60 days, number of branches per plant 30 days, number of branches per plant 60 days, number of pods per plant, number of seeds per pod, 1000 seed weight, biological yield per plant $(\mathrm{g})$, harvest index, seed yield per plant $(\mathrm{g})$, seed germination $\%$, seedling length and vigour index.

\section{Results and Discussion}

The progress in plant breeding depends upon effective selection scheme based on the correlated and non-correlated response. The seed yield or economic yield in almost all the crops is referred as super character which results from the multiplicative interactions of several other characters which are termed as yield components.

Thus, identification of important yield components and information about their association with seed yield and also with each other is very useful for selecting efficient genotypes for evolving high yielding varieties. In this respect, the correlation coefficient which provides symmetrical measurement of degree of association between two variables or characters, help us in understanding the nature and magnitude of association among yield and yield components. The phenotypic correlation coefficients are presented in table 1.

\section{Simple association coefficient}

The estimates of association coefficients among 13 quantitative characters estimated during 2015-16 and 2016-17 are given in Table 4.17 and 4.18, respectively. Estimates of simple association coefficients among 13 quantitative characters estimated during 201617 displayed that seed yield per plant exhibited highly positive significant associations with vigour index (0.980), biological yield per plant (1.000), seedling length (0.958), seed germination (0.967), 
harvest index (0.931), 1000 seed weight (0.942), number of seeds per pod (0.980), number of pods per plant (0.967), No. of branches/plant 60 days (0.967), No. of branches/plant 30 days (0.980), plant height (cm) 60 days $(0.968)$, plant height $(\mathrm{cm}) 30$ days (0.980), days to maturity (0.941) and days to $50 \%$ flowering $(0.941)$. All the other characters under study also displayed highly significant associations with each other besides seed yield per plant. Similar results were also exerted by Johnson et al., (1955), Younis (2008), Karadavut et al., (2009), Azizi-Chakherchaman (2009) and Tyagi, (2010).

Table.1 Treatments used in this study

\begin{tabular}{|c|c|}
\hline Treatment No. & Description \\
\hline $\mathrm{T}_{0}$ & Control (without any biofortification or seed priming) \\
\hline $\mathrm{T}_{1}$ & $\mathrm{GA}_{3} 50 \mathrm{ppm}$ (priming) \\
\hline $\mathrm{T}_{2}$ & $\mathrm{GA}_{3} 100 \mathrm{ppm}$ (priming) \\
\hline $\mathrm{T}_{3}$ & Kinetin 50 ppm (priming) \\
\hline $\mathrm{T}_{4}$ & Kinetin100 ppm (priming) \\
\hline $\mathrm{T}_{5}$ & Kinetin150 ppm (priming) \\
\hline $\mathrm{T}_{6}$ & NAA 50 ppm (priming) \\
\hline $\mathrm{T}_{7}$ & NAA 100 ppm (priming) \\
\hline $\mathrm{T}_{8}$ & seed priming with GA $\mathrm{ppm}_{3} 50 \mathrm{ppm}+$ Foliar spray \\
\hline $\mathrm{T}_{9}$ & seed priming with GA $3100 \mathrm{ppm}+$ Foliar spray \\
\hline $\mathrm{T}_{10}$ & seed priming with GA 3 150ppm +Foliar spray \\
\hline$T_{11}$ & seed priming with Kinetin 50ppm +Foliar spray \\
\hline $\mathrm{T}_{12}$ & seed priming with Kinetin 100ppm +Foliar spray \\
\hline $\mathrm{T}_{13}$ & seed priming with Kinetin 150ppm +Foliar spray \\
\hline $\mathrm{T}_{14}$ & seed priming with NAA 50ppm +Foliar spray \\
\hline $\mathrm{T}_{15}$ & seed priming with NAA 100ppm +Foliar spray \\
\hline $\mathrm{T}_{16}$ & seed priming with NAA 150ppm +Foliar spray \\
\hline $\mathrm{T}_{17}$ & \\
\hline $\mathrm{T}_{18}$ & \\
\hline
\end{tabular}


Table.2 Estimates of correlation coefficients of different characters with seed yield per plant (2015-16)

\begin{tabular}{|c|c|c|c|c|c|c|c|c|c|c|c|c|c|c|}
\hline Character & $\begin{array}{l}\text { Days to } \\
\text { maturity }\end{array}$ & $\begin{array}{c}\text { Plant } \\
\text { height } \\
\text { (cm) } 30 \\
\text { days }\end{array}$ & $\begin{array}{c}\text { Plant } \\
\text { height } \\
\text { (cm) } 60 \\
\text { days }\end{array}$ & $\begin{array}{c}\text { No. of } \\
\text { branches/ } \\
\text { plant } 30 \\
\text { days }\end{array}$ & $\begin{array}{c}\text { No. of } \\
\text { branches } \\
\text { /plant } 60 \\
\text { days }\end{array}$ & $\begin{array}{c}\text { No. of } \\
\text { pods/plant }\end{array}$ & $\begin{array}{c}\text { No. of } \\
\text { seeds/pod }\end{array}$ & $\begin{array}{c}\text { 1000- } \\
\text { seed } \\
\text { weight } \\
(\mathrm{g})\end{array}$ & $\begin{array}{l}\text { Biological } \\
\text { yield (g) }\end{array}$ & $\begin{array}{c}\text { Harvest } \\
\text { index }\end{array}$ & $\begin{array}{c}\text { Seed } \\
\text { germinatio } \\
\mathbf{n}(\%)\end{array}$ & $\begin{array}{l}\text { Seedlin } \\
\text { g length } \\
(\mathrm{cm})\end{array}$ & $\begin{array}{l}\text { Vigour } \\
\text { index }\end{array}$ & $\begin{array}{l}\text { Seed } \\
\text { yield/plant } \\
\text { (g) }\end{array}$ \\
\hline $\begin{array}{c}\text { Days to } 50 \% \\
\text { flowering }\end{array}$ & $0.990 * *$ & $0.909 * *$ & $0.955 * *$ & $0.909 * *$ & $0.954 * *$ & $0.954 * *$ & $0.909 * *$ & $0.953 * *$ & $0.941 * *$ & $0.969 * *$ & $0.954 * *$ & $0.980 * *$ & $0.967 * *$ & $0.941 * *$ \\
\hline Days to maturity & 1 & $0.909 * *$ & $0.955^{* *}$ & $0.909 * *$ & $0.954 * *$ & $0.954 * *$ & $0.909 * *$ & $0.953 * *$ & $0.941 * *$ & $0.969 * *$ & $0.954 * *$ & $0.980 * *$ & $0.967 * *$ & $0.941 * *$ \\
\hline $\begin{array}{c}\text { Plant height }(\mathrm{cm}) \\
30 \text { days }\end{array}$ & & 1 & $0.909 * *$ & $0.923 * *$ & $0.907 * *$ & $0.907 * *$ & $0.988 * *$ & $0.885^{* *}$ & $0.979 * *$ & $0.942 * *$ & $0.907 * *$ & $0.950 * *$ & $0.931 * *$ & $0.980 * *$ \\
\hline $\begin{array}{c}\text { Plant height }(\mathrm{cm}) \\
60 \text { days }\end{array}$ & & & 1 & $0.909 * *$ & $1.000 * *$ & $1.000 * *$ & $0.909 * *$ & $0.958 * *$ & $0.969 * *$ & $0.905 * *$ & $1.000 * *$ & $0.941 * *$ & $0.997 * *$ & $0.968 * *$ \\
\hline $\begin{array}{c}\text { No. of } \\
\text { branches/plant } \\
30 \text { days }\end{array}$ & & & & 1 & $0.907 * *$ & $0.907 * *$ & $0.988 * *$ & $0.885^{* *}$ & $0.979 * *$ & $0.942 * *$ & $0.907 * *$ & $0.950 * *$ & $0.931 * *$ & $0.980^{* *}$ \\
\hline $\begin{array}{c}\text { No. of } \\
\text { branches/plant } \\
60 \text { days }\end{array}$ & & & & & 1 & $0.946 * *$ & $0.907 * *$ & $0.962 * *$ & $0.968 * *$ & $0.902 * *$ & $0.990 * *$ & $0.940 * *$ & $0.997 * *$ & $0.967 * *$ \\
\hline No. of pods/plant & & & & & & 1 & $0.907 * *$ & $0.962 * *$ & $0.968 * *$ & $0.902 * *$ & $0.965 * *$ & $0.940 * *$ & $0.997 * *$ & $0.967 * *$ \\
\hline No. of seeds/pod & & & & & & & 1 & $0.885^{* *}$ & $0.979 * *$ & $0.942 * *$ & $0.907 * *$ & $0.950 * *$ & $0.931 * *$ & $0.980 * *$ \\
\hline $\begin{array}{c}\text { 1000-seed weight } \\
\text { (g) }\end{array}$ & & & & & & & & 1 & $0.943 * *$ & $0.904 * *$ & $0.962 * *$ & $0.945 * *$ & $0.967 * *$ & $0.942 * *$ \\
\hline $\begin{array}{c}\text { Biological yield } \\
\text { (g) }\end{array}$ & & & & & & & & & 1 & $0.929 * *$ & $0.968 * *$ & $0.957 * *$ & $0.980 * *$ & $1.000 * *$ \\
\hline Harvest index & & & & & & & & & & 1 & $0.902 * *$ & $0.982 * *$ & $0.926 * *$ & $0.931 * *$ \\
\hline $\begin{array}{c}\text { Seed germination } \\
(\%)\end{array}$ & & & & & & & & & & & 1 & $0.940 * *$ & $0.997 * *$ & $0.967 * *$ \\
\hline $\begin{array}{l}\text { Seedling length } \\
(\mathrm{cm})\end{array}$ & & & & & & & & & & & & 1 & $0.961 * *$ & $0.958 * *$ \\
\hline Vigour index & & & & & & & & & & & & & 1 & $0.980 * *$ \\
\hline
\end{tabular}


Table.3 Estimates of correlation coefficients of different characters with seed yield per plant (2016-17)

\begin{tabular}{|c|c|c|c|c|c|c|c|c|c|c|c|c|c|c|}
\hline Character & $\begin{array}{l}\text { Days to } \\
\text { maturity }\end{array}$ & $\begin{array}{c}\text { Plant } \\
\text { height } \\
\text { (cm) } 30 \\
\text { days }\end{array}$ & $\begin{array}{c}\text { Plant } \\
\text { height } \\
(\mathrm{cm}) 60 \\
\text { days }\end{array}$ & $\begin{array}{c}\text { No. of } \\
\text { branches/ } \\
\text { plant } 30 \\
\text { days }\end{array}$ & $\begin{array}{c}\begin{array}{c}\text { No. of } \\
\text { branche }\end{array} \\
\text { s/plant } \\
60 \text { days }\end{array}$ & $\begin{array}{c}\text { No. of } \\
\text { pods/plant }\end{array}$ & $\begin{array}{c}\text { No. of } \\
\text { seeds/pod }\end{array}$ & $\begin{array}{l}\text { 1000-seed } \\
\text { weight (g) }\end{array}$ & $\begin{array}{c}\text { Biological } \\
\text { yield (g) }\end{array}$ & $\begin{array}{c}\text { Harvest } \\
\text { index }\end{array}$ & $\begin{array}{c}\text { Seed } \\
\text { germination } \\
(\%)\end{array}$ & $\begin{array}{l}\text { Seedling } \\
\text { length } \\
(\mathrm{cm})\end{array}$ & $\begin{array}{l}\text { Vigour } \\
\text { index }\end{array}$ & $\begin{array}{l}\text { Seed } \\
\text { yield/plan } \\
t(g)\end{array}$ \\
\hline $\begin{array}{c}\text { Days to } 50 \% \\
\text { flowering }\end{array}$ & $0.978 * *$ & $0.909 * *$ & $0.952^{* *}$ & $0.909 * *$ & $0.952^{* *}$ & $0.952^{* *}$ & $0.909 * *$ & $0.956^{* *}$ & $0.942 * *$ & $0.966^{* *}$ & $0.952^{* *}$ & $0.976^{* *}$ & $0.965^{* *}$ & $0.943^{* *}$ \\
\hline Days to maturity & 1 & $0.909 * *$ & $0.952 * *$ & $0.909 * *$ & $0.952^{* *}$ & $0.952^{* * *}$ & $0.909 * *$ & $0.956^{* * *}$ & $0.942 * *$ & $0.966^{* * *}$ & $0.952 * *$ & $0.976 * *$ & $0.965^{* *}$ & $0.943 * *$ \\
\hline $\begin{array}{c}\text { Plant height }(\mathrm{cm}) \\
30 \text { days }\end{array}$ & & 1 & $0.894^{* *}$ & $0.938 * *$ & $0.893^{* *}$ & $0.893^{* *}$ & $0.966^{* *}$ & $0.887^{* *}$ & $0.977 * *$ & $0.944 * *$ & $0.893 * *$ & $0.954 * *$ & $0.922^{* *}$ & $0.978^{* *}$ \\
\hline $\begin{array}{c}\text { Plant height }(\mathrm{cm}) \\
60 \text { days }\end{array}$ & & & 1 & $0.894^{* *}$ & $1.000^{* *}$ & $1.000^{* *}$ & $0.894 * *$ & $0.960^{* *}$ & $0.964 * *$ & $0.896^{* *}$ & $1.000^{* * *}$ & $0.934 * *$ & $0.997 * *$ & $0.963 * *$ \\
\hline $\begin{array}{c}\text { No. of } \\
\text { branches/plant } \\
30 \text { days }\end{array}$ & & & & 1 & $0.893^{* *}$ & $0.893^{* * *}$ & $0.956^{* *}$ & $0.887^{* * *}$ & $0.977 * *$ & $0.944 * *$ & $0.893 * *$ & $0.954 * *$ & $0.922 * *$ & $0.978^{* * *}$ \\
\hline $\begin{array}{c}\text { No. of } \\
\text { branches/plant } \\
60 \text { days }\end{array}$ & & & & & 1 & $0.973^{* *}$ & $0.893 * *$ & $0.961 * *$ & $0.964 * *$ & $0.895^{* *}$ & $0.929 * *$ & $0.934 * *$ & $0.997 * *$ & $0.962^{* *}$ \\
\hline No. of pods/plant & & & & & & 1 & $0.893^{* * *}$ & $0.961^{* * *}$ & $0.964 * *$ & $0.895^{* * *}$ & $0.929 * *$ & $0.934 * *$ & 0.997 ** & $0.962^{\text {*** }}$ \\
\hline No. of seeds/pod & & & & & & & 1 & 0.887 *** & $0.977 * *$ & $0.944 * *$ & $0.893 * *$ & $0.954 * *$ & $0.922^{* *}$ & $0.978 * *$ \\
\hline $\begin{array}{c}\text { 1000-seed weight } \\
\text { (g) }\end{array}$ & & & & & & & & 1 & $0.945^{* *}$ & $0.905^{* *}$ & $0.961^{* *}$ & $0.945^{* *}$ & $0.966^{* *}$ & $0.944 * *$ \\
\hline $\begin{array}{c}\text { Biological yield } \\
\text { (g) }\end{array}$ & & & & & & & & & 1 & $0.930 * *$ & $0.964 * *$ & $0.959 * *$ & $0.978^{* *}$ & $1.000^{* *}$ \\
\hline Harvest index & & & & & & & & & & 1 & $0.895^{* *}$ & $0.981 * *$ & $0.922 * *$ & $0.932 * *$ \\
\hline $\begin{array}{c}\text { Seed germination } \\
(\%)\end{array}$ & & & & & & & & & & & 1 & $0.934 * *$ & $0.997 * *$ & $0.962^{* *}$ \\
\hline $\begin{array}{l}\text { Seedling length } \\
(\mathrm{cm})\end{array}$ & & & & & & & & & & & & 1 & $0.957^{* *}$ & $0.960^{* * *}$ \\
\hline Vigour index & & & & & & & & & & & & & 1 & $0.977 * *$ \\
\hline
\end{tabular}


Estimates of simple association coefficients among 13 quantitative characters estimated during 2016-17 displayed that seed yield per plant exhibited highly positive significant associations with biological yield per plant (1.000), vigour index (0.977), number of seeds per pod (0.978), seedling length (0.960), seed germination (0.962), harvest index (0.932), 1000 seed weight $(0.944)$, number of pods per plant (0.962), No. of branches/plant 60 days (0.962), No. of branches/plant 30 days (0.978), plant height $(\mathrm{cm}) 60$ days $(0.963)$, plant height (cm) 30 days (0.978), days to maturity (0.943) and days to $50 \%$ flowering (0.943).

All the other characters under study also displayed highly significant associations with each other besides seed yield per plant.

Therefore, these characters emerged as most important factors in influencing seed yield in chickpea. The strong positive correlation of seed yield with the characters mentioned above has also been reported earlier in chickpea by Karadavut (2009).

Biological yield per plant, pods per plant, harvest index, plant height and 100 seed weight had strong positive associations with one another besides, having strong positive association with seed yield per plant, as earlier reported by Naresh et al., (2009), Al-Ghazawi et al., 2011, Azizi-Chakherchaman et al., (2009). Furthermore; days to 50 per cent flowering, days to maturity, plant height, secondary branches per plant, pods per plant were strongly associated with each other and these traits add towards higher biomass. These findings are broadly in agreement with some of the earlier reports of Younis et al., (2009) and Karadavut et al., (2009).

\section{References}

Al-Ghazawi, A.L.A., Bsoul, E., Ankour, F., AlAjlouni, Z., Al- Azzam, M. and Ajlouni, M.M. 2011. Genetic variability for quantitative traits in Jordanian lentil; Advances in Environmental Biology, 5(11); 3676-3680.

Anonymous, 2013. Agriculture Statistic Division, Directorate of Economics and Statistics, Department of Agriculture and Cooperation, U.P.

Azizi-Chakherchaman, S., Mostafaei, H., Hassanpanah, D., Kazemi-Arbat, H. and Yarnia, M. 2009. Path coefficient analysis of yield and yield components in promising lentil (Lens culinaris L.) genotypes under dry land conditions. J. of New Agricultural Sciences. 5 (17): 45-56.

Johnson, H.W., Robinson, H.F. and Comstock, R.E. 1955. Estimates of phenotypic and genotypic correlation in soybean and their implication in selection. Agronomic Journal, 47: 477-482.

Karadavut, U., 2009. Path analysis for yield and yield components in lentil (Lens culinaris Medik.). Turkish J. of Field Crops, 14 (2): 97-104.

Naresh Chahota, R.K., and Sood, B.C. 2009. Component analysis for seed yield and yield traits in micro-sperma $x$ macrosperma derivatives of lentil (Lens culinaris Medik.). Agricultural Science Digest. 29 (3): 163-168.

Searle, S.R., 1961. Phenotypic, genotypic and environmental correlations. Biometrics, 17: 474-480.

Tyagi, S.D., and Khan, M.H. 2010. Genetic divergence in lentil. African Crop Science J., 18 (2): 69-74.

Younis, N., Hanif, M., Siddique, S., Abbas, G., Asghar, M.J. and Haq, M.A. 2008. Estimates of genetic parameter and path analysis in lentil (Lens culinaris Medik.). Pak. J. Agric. Sci., Vol. 45 (3): 45-48.

\section{How to cite this article:}

Pankaj Kumar, S.C. Vimal and Ajay Kumar. 2017. Study of Simple Correlation Coefficients for Yield and its Component Traits in Lentil (Lens culinaris Medik.). Int.J.Curr.Microbiol.App.Sci. 6(9): 32603265. doi: https://doi.org/10.20546/ijcmas.2017.609.401 